\title{
Asymptomatic Subclinical Cases of Coronavirus Disease 2019 without Viral Transmission in Three Independent Families
}

This article was published in the following Dove Press journal:

Infection and Drug Resistance

\author{
Xian Zhang ${ }^{\mathrm{l}, *}$ \\ Liting Chen ${ }^{2 * *}$ \\ Jia $\mathrm{Wei}^{2}$ \\ Jianfeng Zhou ${ }^{2}$ \\ Yang $\mathrm{CaO}^{2}$ \\ Gaoxiang Wang ${ }^{2}$ \\ 'Department of Ophthalmology, Tongji \\ Hospital, Tongji Medical College, \\ Huazhong University of Science and \\ Technology, Wuhan, Hubei 430030, \\ People's Republic of China; ${ }^{2}$ Department \\ of Hematology, Tongji Hospital, Tongji \\ Medical College, Huazhong University of \\ Science and Technology, Wuhan, Hubei \\ 430030, People's Republic of China \\ *These authors contributed equally to \\ this work
}

Purpose: There is increasing evidence indicating that considerable fractions of cases of SARS-CoV-2 infection are asymptomatic. We traced three asymptomatic clusters to investigate the infectivity of subclinical cases of coronavirus disease 2019 (COVID-19).

Patients and Methods: Three medical staff who were asymptomatic were diagnosed with coronavirus disease 2019 by serological tests. Their close contacts were systematically evaluated based on COVID-19-related symptoms, nucleic acid tests, serological tests, and chest computed tomography (CT) as needed to determine if they were infected by SARSCoV-2.

Results: None of the staff's close contacts, including 10 family members, were infected by the indexes, even though no protective measures were taken.

Conclusion: The infectivity of asymptomatic subclinical infection patients of coronavirus disease 2019 seems to be low.

Keywords: SARS-CoV-2, COVID-19, asymptomatic, close contact, infectivity

\section{Introduction}

Toward the end of 2019, there was an outbreak of severe acute respiratory syndrome coronavirus 2 (SARS-CoV-2). This led to coronavirus disease 2019 (COVID-19) — which rapidly spread throughout the world, causing a pandemic. ${ }^{1,2}$ As of May 5, 2020, there were over 35,00,000 confirmed COVID-19 cases, with more than 250,000 deaths globally. Currently, increasing evidence indicates that a considerable fraction of people infected with SARS-CoV-2 is asymptomatic. These people are often hidden in the population, raising concerns about their infectiousness.

\section{Patients and Methods}

Three medical staff diagnosed with asymptomatic SARS-CoV-2 infection by serological tests after returning to work and their family members were recruited for this study. The study was approved by the Ethics Committee of Tongji Hospital, Tongji Medical College, Huazhong University of Science and Technology (IRB ID: TJ-C20200114) and was conducted according to the principles of the Declaration of Helsinki. Written informed consent was obtained from patients and their family members to conduct the study and to have case details and associated images published. The patients and their close contacts were systematically evaluated
Correspondence: Gaoxiang Wang; Yang Cao

Department of Hematology, Tongji Hospital, Tongji Medical College, Huazhong University of Science and

Technology, Wuhan, Hubei 430030,

People's Republic of China

$\mathrm{Tel} / \mathrm{Fax}+86$ 27-83662680

Email gxwtjxy@I26.com;

caoyangemma@163.com 
based on COVID-19-related symptoms, nucleic acid tests, serological tests, and chest computed tomography (CT) as needed to determine if they were infected with SARSCoV-2. COVID-19 diagnosis was made according to the guideline presented in Novel Coronavirus Infection Pneumonia Diagnosis and Treatment Guideline (7th edition), published by the National Health Commission of China. $^{3}$

\section{Detection of SARS-CoV-2 by Conventional Real-Time PCR}

SARS-CoV-2 RNA was detected by real-time PCR, as reported in a previous study, using the diagnostic criteria recommended by the National Center for Disease Control and Prevention (CDC) (China). ${ }^{4}$ A test producing a cycle threshold $(\mathrm{Ct})$ value less than 37 was defined as a positive test, while a test producing a $\mathrm{Ct}$ value of 40 or more was defined as a negative test. A medium load, defined as a $\mathrm{Ct}$ value of 37 to less than 40, required confirmation by at least two repeated tests.

\section{Serologic Tests for Anti-SARS-CoV-2 Specific Antibodies}

SARS-CoV-2-specific IgM and IgG were tested by paramagnetic particle chemiluminescent immunoassay using iFlashSARS-CoV-2 IgM/IgG assay kit (Shenzhen YHLO Biotech
Co., Ltd) and iFlash Immunoassay Analyzer (Shenzhen YHLO Biotech Co., Ltd). The specificity and sensitivity of SARS-CoV-2 IgM and IgG detection were also evaluated (See Supplementary method). The results were similar to those in other studies. ${ }^{5}$

\section{Chest Computer Tomography Scanning}

Patients were scanned by noncontract-enhanced chest CT examination in the supine position during end-inspiration, ranging from the apex to the base of the lung. Image reconstruction was performed with a slice thickness of $1.25 \mathrm{~mm}$.

\section{Results}

Three medical staff were accidentally discovered to be serologically positive (IgG positive and $\operatorname{IgM}$ negative) after returning to work on April 3, 2020, suggesting that they had been previously infected with SARS-CoV-2. All three staff denied having any symptoms of COVID-19 (including fever, cough, and diarrhea). Till the time of writing this report, the $\mathrm{CT}$ images were completely normal for Cases 1 and 2 (Figure 1A and B), whereas Case 3 showed a slight single sub-pleural patch on the left side (Figure 1C). No viral shedding was detected in the swabs of the three patients. Since the outbreak of this disease, all three patients have been living with their family members

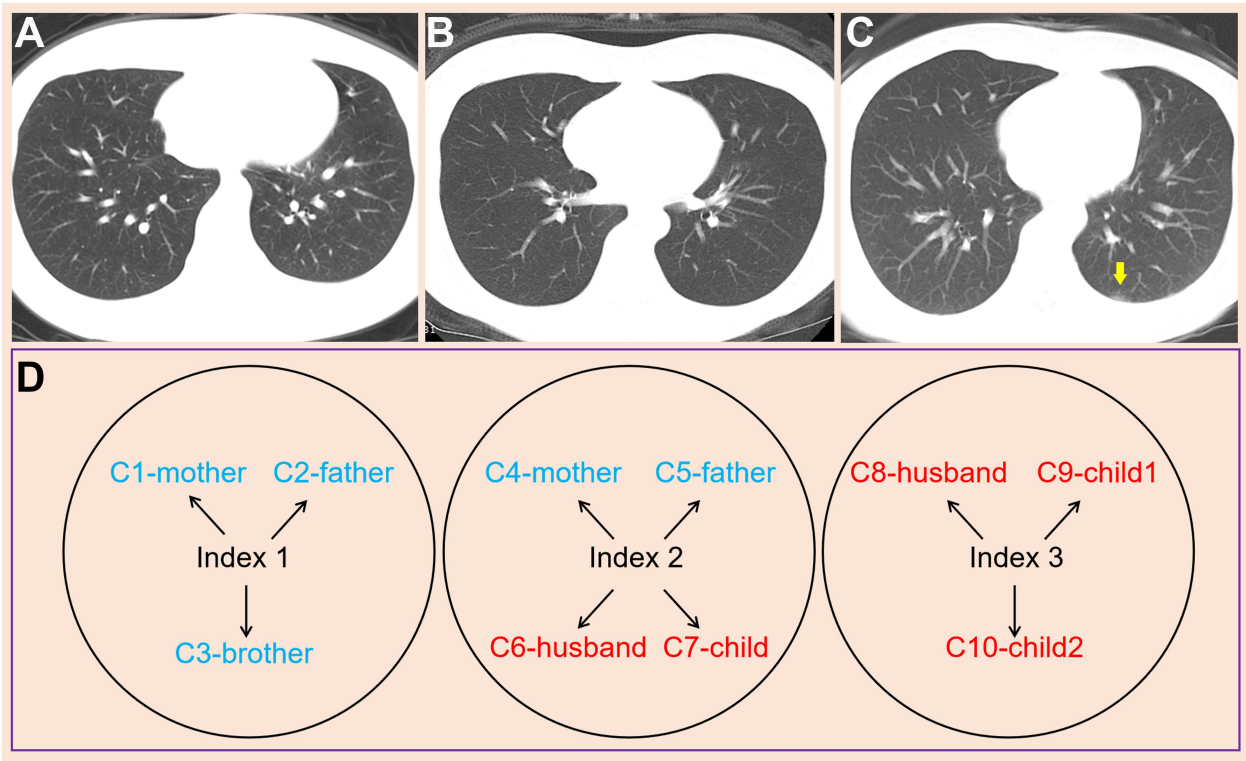

Figure I Chest computed tomography performance of the indexes and contact tracing investigation of their contacts. (A and B) completely normal computed tomography of Case I and Case 2; (C) a slight single subpleural patch on the left side of Case 3 (yellow arrow); (D) contact tracing investigation of three clusters. The blue color indicates contacts living with indexes in the same apartment, eating together but sleeping in different rooms; the red color indicates persons with the closest contact with the indexes-living in the same apartment, eating together, and sleeping in the same rooms. None of the contacts were infected, according to their symptoms and the nucleic acid and serologic tests. 
without taking any protective measures. All their family members - including four old people, three young persons, and three children-showed no symptoms of COVID-19, and their nucleic acid and antibody tests were negative, indicating that they were not infected.

\section{Case Presentation}

Case 1 presents a nurse at the hematology department who revealed that she might have had contact with a patient around January 25 that was later diagnosed with COVID19 pneumonia on January 28, 2020. However, because she experienced no signs or symptoms after a few months, she neglected the suspicious exposure history and did not isolate herself from her parents and younger brother.

Case 2 presents a nurse at the ophthalmology department who claimed that she performed a venipuncture without any personal protective equipment in January on a sick colleague that later became symptomatic and was diagnosed with COVID-19. She denied having symptoms such as fever, cough, and diarrhea but admitted she had a slight backache two days after she had contact with the sick colleague. She thought the backache, which relieved spontaneously, was caused by her wrong sleeping position. Moreover, she ignored her exposure history probably on January 17 , and continued living with her parents, husband, and child without taking any protective measures.
Case 3 presents a hospital finance staff who was not sure about her exposure history but had muscle soreness without fever, cough, and diarrhea in the end of January. In her memory, the most possible exposure should be the experience of taking the subway to work during January 16-20 mornings. Her muscle soreness also recovered spontaneously within a few days without any intervention while she was living with her husband and two children.

\section{Contact Tracing Investigation}

The family members of the three indexes-including their parents, husbands, children, and siblings - were evaluated for COVID-19 infection. Among these 10 close contacts, there were 4 old people, 3 young persons, and 3 children who had been living together with the indexes in the same apartments without any protection since the outbreak of COVID-19 (Figure 1D). We inquired about their clinical features over the past few months, and none of the family members showed clinical presentations of COVID-19. In addition, both their nasopharyngeal swabs for SARS-CoV-2 Real-Time-PCR and their serologic tests were negative. Taken together, all the intimate family contacts were not infected, including a 48-year-old patient who was newly diagnosed with nasopharyngeal carcinoma and was undergoing radiotherapy and chemotherapy (Table 1).

Table I Evaluation of COVID-I9 Infection of Three Clusters

\begin{tabular}{|c|c|c|c|c|c|c|c|c|c|}
\hline & \multirow[t]{2}{*}{ Gender } & \multirow[t]{2}{*}{ Age } & \multirow[t]{2}{*}{ Relationship } & \multirow[t]{2}{*}{ Comorbidities } & \multirow[t]{2}{*}{$\begin{array}{l}\text { Clinical } \\
\text { Presentations }\end{array}$} & \multirow[t]{2}{*}{$\begin{array}{l}\text { CT } \\
\text { Image }\end{array}$} & \multirow[t]{2}{*}{$\begin{array}{l}\text { Detection of } \\
\text { Swabs }\end{array}$} & \multicolumn{2}{|c|}{$\begin{array}{l}\text { Serological } \\
\text { Tests }^{\text {a }} \text { (AU/ } \\
\text { mL) }\end{array}$} \\
\hline & & & & & & & & IgM & IgG \\
\hline Index I & $\mathrm{F}$ & 26 & & No & No & Normal & - & - & 81.13 \\
\hline $\mathrm{Cl}$ & $\mathrm{F}$ & 46 & Mother & No & No & NA & - & - & - \\
\hline $\mathrm{C} 2$ & $M$ & 48 & Father & NPC & No & NA & - & - & - \\
\hline $\mathrm{C} 3$ & M & 19 & Brother & No & No & NA & - & - & - \\
\hline Index2 & $\mathrm{F}$ & 30 & & No & Backache & Normal & - & - & 106.05 \\
\hline $\mathrm{C} 4$ & $\mathrm{~F}$ & 53 & Mother & No & No & NA & - & - & - \\
\hline $\mathrm{C} 5$ & $M$ & 55 & Father & No & No & NA & - & - & - \\
\hline $\mathrm{C} 6$ & M & 32 & Husband & No & No & NA & - & - & - \\
\hline $\mathrm{C7}$ & M & 4 & Son & No & No & NA & - & - & - \\
\hline Index3 & $\mathrm{F}$ & 36 & & No & Muscle soreness & Abnormal & - & - & 79.02 \\
\hline $\mathrm{C} 8$ & M & 37 & Husband & No & No & NA & - & - & - \\
\hline C9 & $\mathrm{F}$ & 10 & Daughter & No & No & NA & - & - & - \\
\hline $\mathrm{ClO}$ & $M$ & 3 & Son & No & No & NA & - & - & - \\
\hline
\end{tabular}

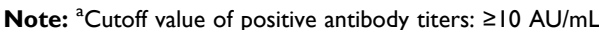

Abbreviations: F, female; M, male; NPC, nasopharyngeal carcinoma; NA, not available. 


\section{Discussion}

It is well known that symptomatic patients of COVID-19 can transmit the SARS-CoV-2 viruses in direct (respiratory aerosols or droplets) and indirect (fecal-oral, hands-eye) manners. ${ }^{6-9}$ Physical distancing, face masks, and eye protection are all recommended for preventing person-to-person transmission of SARS-CoV-2. ${ }^{10}$

While Dr. Zhang and his colleagues noted that asymptomatic COVID-19 exists and should be actually regarded as a subclinical infection. ${ }^{11}$ It is important to be distinguished from other similar conditions, such as pre-symptomatic in the incubation period and chronic asymptomatic carrier like Typhoid Mary. All the patients in our study met the diagnostic criteria for subclinical infection.

In general, patients with a history of exposure, regardless of clinical or radiation performance, should be placed under medical observation and isolation from their family according to the current policy. Therefore, there is little opportunity to deliberately observe whether asymptomatic patients can infect the surrounding contacts under natural conditions. Intriguingly, the cases in this study provide an extreme situation of natural exposure. All of them unintentionally exposed themselves in the mid-January. During the following 2 months more, almost covering the whole disease process, from the incubation period to the recovery period, the indexes lived together with their family members, including a nasopharyngeal carcinoma patient who is theoretically vulnerable to SARS-CoV-2 infection due to impaired immune function as a result of radiotherapy or chemotherapy, without taking any protective measures. Surprisingly, according to the epidemiological and clinical data of the three family clusters, none of their family members were infected. This suggests that, even in close contact, the infectivity of asymptomatic subclinical infections may be relatively low.

Why asymptomatic subclinical infection of COVID-19 seems to be less infectious is still not very clear. The patients in our study had no fever, no cough nor any other respiratory symptoms, and also had normal or almost normal chest CT. Two of the patients had low back pain and muscle soreness, which may be consistent with the manifestations of viremia, rather than the signs of lung infections. Thus, we speculate that such subclinical patients may have transient viremia. Chest CT showed no or slight lesion in the patients' lungs, indicating that the replication and excretion of the virus from the lungs is very low and thus resulting in low infectivity. Our findings are consistent with another investigation by Scott and his colleagues, who reported that patients with a mildly symptomatic case did not transmit the infection to their contacts. ${ }^{12}$ In another study, Zou and his colleagues reported that SARS-CoV-2 viral shedding in asymptomatic cases was similar to those detected in symptomatic patients, which indicates that asymptomatic patients have the potential to transmit the virus. However, their study only involved one asymptomatic patient so they could not effectively observe whether the asymptomatic patient was actually responsible for the transmission of SARS-CoV-2. ${ }^{13}$ Therefore, our perspective agrees that asymptomatic subclinical infections are less infectious and further studies are needed to determine the transmission dynamics of subclinical infections.

At present, with the development of extensive screening, more asymptomatic people have been diagnosed. With the social and economic pressure, nonselective isolation and social interruption for a considerable number of asymptomatic patients will have a great negative impact on people's daily life and the public health expenditure. ${ }^{14}$ Therefore, based on our preliminary findings, we suggest that large-scale epidemiological investigation should be carried out to carefully evaluate the infectivity of the virus and its related factors in asymptomatic patients. By carefully assessing the risk of infectivity, we advocate to develop refined prevention and control strategies that can help to normalize our social-economic life and save more public health costs.

\section{Conclusion}

In conclusion, our study is the first to assess infectivity of the asymptomatic subclinical cases of COVID-19. We found that the transmission of asymptomatic subclinical cases seems low, which may be due to the lack of upper respiratory symptoms as well as zero or extremely mild involvement of the lungs.

\section{Acknowledgments}

We are grateful to Weiyong Liu and Huijun Li, the laboratory staffs, for their efforts regarding detecting swabs by RT-PCR and serologic tests for specific antibodies against SARS-CoV-2.

\section{Funding}

This work was supported by Emergency Research Project of Tongji hospital of Huazhong University of Science and Technology (Grant Number: 2020kfyXGYJ045, to Dr. JZ). 


\section{Disclosure}

The authors report no conflicts of interest in this work.

\section{References}

1. Li Q, Guan X, Wu P, et al. Early transmission dynamics in Wuhan, China, of novel coronavirus-infected pneumonia. $N$ Engl J Med. 2020;382(13):1199-1207. doi:10.1056/NEJMoa2001316

2. Mahase E. Covid-19: WHO declares pandemic because of "alarming levels" of spread, severity, and inaction. BMJ. 2020;368:m1036. doi:10.1136/bmj.m1036

3. National Health Commission of the people's republic of China. Pneumonia diagnosis and treatment of novel coronavirus infection (Pilot version 7). 2020.

4. Ai T, Yang Z, Hou H, et al. Correlation of chest CT and RT-PCR testing in coronavirus disease 2019 (COVID-19) in China: a report of 1014 cases. Radiology. 2020;26:200642.

5. Qian C, Zhou M, Cheng F, et al. Development and multicenter performance evaluation of the first fully automated SARA-CoV-2 IgM and IgG immunoassays. MedRxiv. 2020. doi:10.1101/2020.04. 16.20067231

6. Meselson M. Droplets and aerosols in the transmission of SARS-CoV2. $N$ Engl J Med. 2020.

7. Yeo C, Kaushal S, Yeo D. Enteric involvement of coronaviruses: is faecal-oral transmission of SARS-CoV-2 possible? Lancet Gastroenterol Hepatol. 2020;5(4):335-337. doi:10.1016/S2468-1253 (20)30048-0
8. Zhang X, Chen X, Chen L, et al. The evidence of SARS-CoV-2 infection on ocular surface. Ocul Surf. 2020;18(3):360-362. doi:10.1016/j.jtos.2020.03.010

9. Chen L, Deng C, Chen X, et al. Ocular manifestations and clinical characteristics of 535 cases of COVID-19 in Wuhan, China: a crosssectional study. Acta Ophthalmol. 2020. doi:10.1111/aos.14472

10. Maclntyre CR, Wang Q. Physical distancing, face masks, and eye protection for prevention of COVID-19. Lancet. 2020;395 (10242):1950-1951. doi:10.1016/S0140-6736(20)31183-1

11. Zhou X, Li Y, Li T, Zhang W. Follow-up of the asymptomatic patients with SARS-CoV-2 infection. Clin Microbiol Infect. 2020; pii: S1198-743X (20)30169-5.

12. Scott SE, Zabel K, Collins J, et al. First mildly ill, non-hospitalized case of coronavirus disease 2019 (COVID-19) without viral transmission in the United States-Maricopa County, Arizona. Clin Infect Dis. 2020;pii: ciaa374.

13. Zou L, Ruan F, Huang M, et al. SARS-CoV-2 viral load in upper respiratory specimens of infected patients. $N$ Engl J Med. 2020;382 (12):1177-1179. doi:10.1056/NEJMc2001737

14. Hodge JG, Rutkow L, Corcoran AJ, Corcoran AJ. Mental and behavioral health legal preparedness in major emergencies. Public Health Rep. 2010;125(5):759-762. doi:10.1177/003335491012500519
Infection and Drug Resistance

\section{Publish your work in this journal}

Infection and Drug Resistance is an international, peer-reviewed openaccess journal that focuses on the optimal treatment of infection (bacterial, fungal and viral) and the development and institution of preventive strategies to minimize the development and spread of resistance. The journal is specifically concerned with the epidemiology of

\section{Dovepress}

antibiotic resistance and the mechanisms of resistance development and diffusion in both hospitals and the community. The manuscript management system is completely online and includes a very quick and fair peerreview system, which is all easy to use. Visit http://www.dovepress.com/ testimonials.php to read real quotes from published authors. 